\title{
Natalizumab Discontinuation and Treatment Strategies in Patients with Multiple Sclerosis (MS): A Retrospective Study from Two Italian MS Centers
}

\author{
Marianna Lo Re · Marco Capobianco • Paolo Ragonese • \\ Sabrina Realmuto - Simona Malucchi · Paola Berchialla • \\ Giuseppe Salemi · Antonio Bertolotto
}

To view enhanced content go to www.neurologytherapy-open.com

Received: October 16, 2015 / Published online: December 8, 2015

(C) The Author(s) 2015. This article is published with open access at Springerlink.com

\section{ABSTRACT}

Introduction: Natalizumab

(NTZ)

discontinuation can be followed by multiple sclerosis (MS) disease reactivation. Currently no disease-modifying drug (DMD) has been shown to be able to abolish disease reactivation. The aims of the current study were: (1) to determine the frequency of MS reactivation after NTZ discontinuation; (2) to evaluate predictors of reactivation risk, and (3) to compare the effect of different treatments in reducing this risk.

Methods: Data from 132 patients with MS followed-up for 2 years before NTZ treatment and 1 year after interruption were collected

Electronic supplementary material The online version of this article (doi:10.1007/s40120-015-0038-9) contains supplementary material, which is available to authorized users.

M. Lo Re (凹) · P. Ragonese · S. Realmuto · G. Salemi Department of Experimental Biomedicine and Clinical Neurosciences, University of Palermo, Palermo, Italy

e-mail: marianna.lore@gmail.com

M. Capobianco · S. Malucchi · A. Bertolotto Regional Multiple Sclerosis Centre, San Luigi Gonzaga Hospital, Orbassano, Italy

P. Berchialla

Department of Clinical and Biological Sciences, University of Turin, Torino, Italy from two Italian MS centers and retrospectively evaluated.

Results: Overall, 72 of 132 patients (54.5\%) had relapses after NTZ discontinuation and 60 of 125 patients (48\%), who had magnetic resonance imaging, had radiological reactivation. Rebound was observed in 28 of 132 patients $(21.2 \%)$. A higher number of relapses in the 2 years before NTZ treatment, a longer washout period, and a lower number NTZ infusions correlated with reactivation and rebound. Untreated patients $(n=37)$ had higher clinical and radiological activity and rebound in comparison to patients receiving DMDs. Moreover, a lower risk of relapses was found in patients treated with second-line therapies (NTZ and fingolimod) than in those treated with first-line therapies (interferon beta, glatiramer acetate, teriflunomide, azathioprine). Interestingly, no disease reactivation in off-label treatment (rituximab, autologous hematopoietic stem cell transplantation) was observed.

Conclusion: NTZ discontinuation is a risk for MS reactivation and rebound. An alternative treatment should be promptly resumed mainly in patients with a previous very active disease 
course and with a shorter NTZ therapy. Second-line therapies demonstrate superiority in preventing relapses after NTZ discontinuation.

Keywords: Disease reactivation; First-line therapies; Multiple sclerosis; Natalizumab discontinuation; Rebound; Second-line therapies

\section{INTRODUCTION}

Natalizumab (NTZ) is an effective therapy for reducing disease activity in relapsing-remitting multiple sclerosis (MS) [1, 2]. However, an important safety issue related to its use is progressive multifocal leukoencephalopathy (PML), a demyelinating brain disease triggered by the John Cunningham virus (JCV) [3-5]. NTZ therapy is often discontinued due to the risk of PML; however, this frequently leads to MS disease reactivation. A consistent return of underlying pre-treatment disease activity has been identified by various authors and peaks 4-7 months following NTZ discontinuation [6-13]. The possibility of a rebound effect, where the worsening of disease activity is beyond pre-treatment levels, has also been described in different reports [14-16]. This phenomenon is reported in 10 and $30 \%$ of patients in cohort studies; however, a unique definition has not currently been found $[15,17$, 18].

Several studies have tried to identify the best therapeutic strategy to prevent disease reactivation/rebound after NTZ suspension; however, the optimal alternative treatment regimen has not yet been identified. Disease control has been incomplete when patients switched to interferon beta (IFN $\beta$ ) or glatiramer acetate (GA) [17, 19, 20]. Fingolimod (FTY) may be a reasonable option because of its efficacy. A few observational studies show a reduced annualized relapse rate (ARR) in patients who switched to FTY compared with those who remain untreated or switch to IFN $\beta-1$ a or GA [21-23]. Others studies have reported severe MS relapses and radiologic rebound after the initiation of FTY in patients previously treated with NTZ [24-26]. However, a recent study showed a lower risk of magnetic resonance imaging (MRI) and clinical disease reactivation if FTY therapy is started 8-12 weeks after NTZ discontinuation [27].

In the current study, the clinical and radiological data of patients previously treated with NTZ were collected and analyzed, and then followed-up for 1 year after interruption. The aims of this study were: (1) to determine the frequency of MS reactivation after NTZ discontinuation; (2) to evaluate predictors of reactivation risk, and (3) to compare the effect of different treatments in reducing this risk.

\section{METHODS}

\section{Study Design and Participants}

This retrospective study was conducted at two Italian MS referral centers (Orbassano and Palermo). Data were collected between February 2007 and January 2015 and retrospectively evaluated from electronic databases and clinical records. Radiological and clinical information of patients with MS were analyzed in the 2 years before NTZ treatment and 1 year after NTZ interruption. Ethical approval was not necessary due to the retrospective plan of the study, according to internal ethical board rules. 


\section{Patient Selection for Data Analysis}

We included in our analysis patients with relapsing-remitting MS who received at least 6 infusions of NTZ therapy and with a follow-up of at least 12 months after NTZ discontinuation. Patients who did not meet these inclusion criteria or who developed PML were excluded from the analysis.

\section{Outcome Measures}

Clinical data were collected 2 years before NTZ treatment, and 3, 6, 9, and 12 months after interruption. Clinical MS reactivation was defined as the presence of documented relapses and/or the progression of Expanded Disability Status Scale (EDSS) at the end of follow-up. Radiological MS reactivation was defined as the appearance of new T2+/FLAIR lesions and/or Gd-enhancing lesions. As this was a retrospective study, MRI frequency was not regularly scheduled and so radiological evaluation was done where MRI was available.

The literature lacks a clear and univocal definition of rebound activity. It has been defined as a fatal relapse, or as any radiological or disease activity beyond that seen in the pre-NTZ period. Therefore, an objective definition based on at least two of the following features was arbitrarily decided:

1. An ARR increase in comparison to pre-NTZ disease course;

2. One or more severe relapses with sustained disability progression (one-step EDSS increase);

3. Three or more new large $\mathrm{T} 2$ lesions and/or Gd-enhancing lesions in the MRI;

4. New tumor-like demyelinating lesions in the MRI.

\section{Statistical Analysis}

All statistical analyses were performed using SPSS Statistics version 17.0 (SPSS Inc., Chicago, IL, USA). The correlation between different variables and MS reactivation was analyzed using a univariate statistical analysis: $t$ test for continuous variables and $\chi^{2}$ test for categorical variables. A Cox multivariate regression model was used to minimize the bias effect of unadjusted variables due to the nature of this retrospective analysis. Data were adjusted for number of infusions, relapses in the 2 years before NTZ, washout period between NTZ and new therapy, and therapeutic strategies after NTZ (no therapy, first-line therapies, FTY, NTZ). Statistical significance was defined by a $P$ value $<0.05$. Groups with few patients ( $\leq 8$ patients) were excluded from the analysis (rituximab, immunosuppressive therapy, autologous hematopoietic stem cell transplant [AHSCT]).

\section{RESULTS}

\section{Demographic and Baseline Characteristics}

Of the 346 patients treated with NTZ at the two referral centers, 192 had discontinued treatment: 67 patients from the Palermo MS centre and 125 patients from the Orbassano MS centre. Data from the Orbassano population have been previously described [28]. In particular, the number of patients was lower and with a follow-up ranging between 1 and 12 months after NTZ discontinuation. Subsequently, 57 patients who did not meet the inclusion criteria and three patients who unfortunately developed PML were excluded from the analysis. A total of 132 patents were included in the study (Fig. 1). 


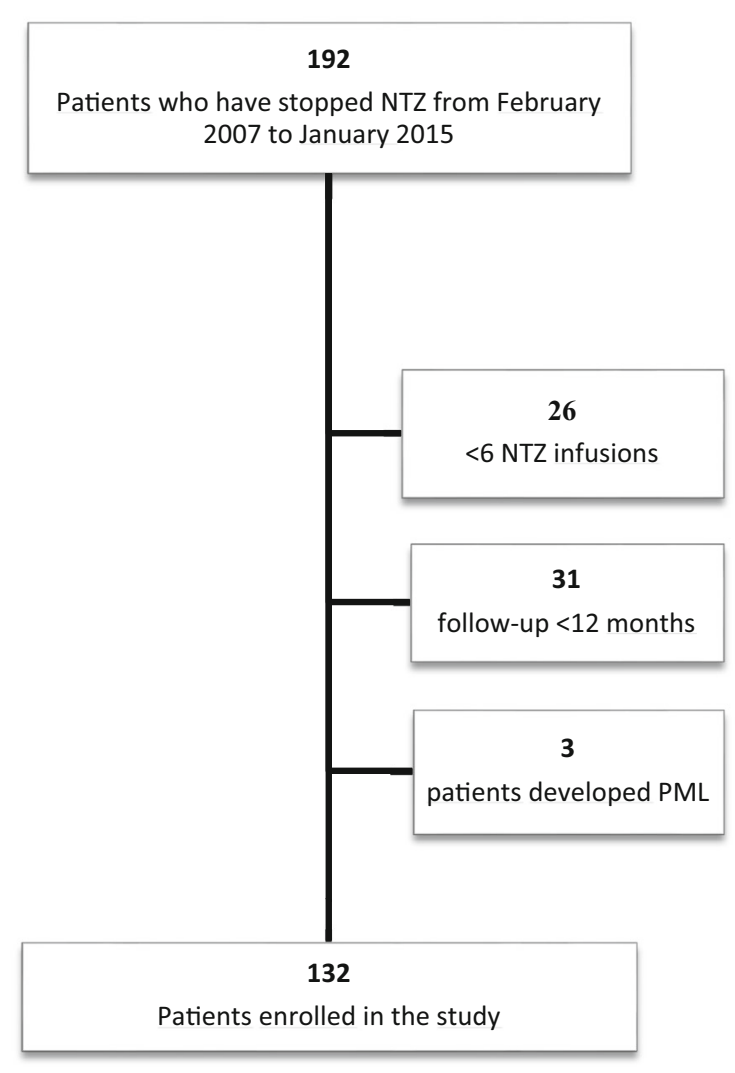

Fig. 1 Enrolment of patients in the study. NTZ natalizumab, $P M L$ progressive multifocal leukoencephalopathy

Of the 132 patients included, 56 were male and 76 were female. The median age at NTZ initiation was 35.4 years (range $29.8-42$ years) and the median EDSS score was 3.5. The median EDSS score 12 months after NTZ discontinuation was 4.0. The median washout time out was 5 months (range $0.3-11$ months). No differences between male and female patients were reported. The primary reason for NTZ interruption was connected to the risk of PML (Table 1).

\section{Disease Reactivation}

Disease reactivation occurred in many patients: 72 of 132 patients (54.5\%) experienced a clinical reactivation. In our analysis 125 of 132 patients performed at least one MRI examination after NTZ discontinuation: 60 out of $125(48 \%)$ presented a radiological reactivation. Furthermore, 28 of 132 patients (21.2\%) had rebound. Clinical and radiological disease activity was most frequent between 6 and 9 months following NTZ discontinuation. A peak in clinical reactivation was found in 7 months after NTZ discontinuation; the frequency of disease reactivation gradually decreased until the end of the follow-up. Similarly, a peak in rebound activity was observed at 7 months, whereas the greater radiological activity was detected at 9 months after NTZ discontinuation.

\section{Effect of Different Treatments}

Ninety-five patients (72\%) were switched to other therapies after a median washout period of 5 months: 57 switched to FTY, 16 to first-line therapies (IFN $\beta$, GA, teriflunomide, azathioprine), 9 to NTZ, 7 to rituximab, 4 to immunosuppressive agents (cyclophosphamide or mitoxantrone), and 2 to AHSCT. Thirty-seven patients (28\%) remained therapy free.

More clinical and radiological reactivation was observed in the therapy-free and first-line therapy groups. One patient treated with mitoxantrone developed a rebound with a strong clinical and radiological reactivation; this was a patient with very aggressive disease, even before and during NTZ treatment. Among patients who switched to FTY, clinical reactivation was observed in 10 patients $(17.5 \%)$ and radiological reactivation was observed in 13 patients (23.6\%). Clinical reactivation and rebound effect was not seen in patients who restarted NTZ; only one patient out of nine who restarted NTZ therapy presented radiological reactivation. 
Table 1 Demographic and baseline characteristics

\begin{tabular}{|c|c|c|}
\hline Characteristics & $N$ & 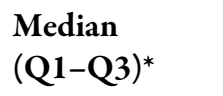 \\
\hline Gender (male/female) & 132 & $56 / 76$ \\
\hline Age (years) & 132 & $41(34.7-47.4)$ \\
\hline Age at onset of NTZ (years) & 132 & $35.4(29.8-42)$ \\
\hline ARR 2 years before NTZ & 129 & $1.0(1.0-1.5)$ \\
\hline \multicolumn{3}{|l|}{ EDSS score } \\
\hline NTZ initiation & 130 & $3.5(2.0-5.5)$ \\
\hline $\begin{array}{l}3 \text { months after NTZ } \\
\text { discontinuation }\end{array}$ & 132 & $4.0(2.0-6.0)$ \\
\hline $\begin{array}{l}6 \text { months after NTZ } \\
\text { discontinuation }\end{array}$ & 132 & $4.0(2.0-6.0)$ \\
\hline $\begin{array}{l}9 \text { months after NTZ } \\
\text { discontinuation }\end{array}$ & 132 & $4.0(2.0-6.0)$ \\
\hline $\begin{array}{l}12 \text { months after NTZ } \\
\text { discontinuation }\end{array}$ & 132 & $4.0(2.0-6.0)$ \\
\hline NTZ infusions & 132 & $25(20.5-38.25)$ \\
\hline ARR 12 months after NTZ & 132 & $1(0-1)$ \\
\hline Washout time (months) & 95 & $5(0.3-11)$ \\
\hline \multicolumn{3}{|c|}{ Primary reason for stopping NTZ (\%) } \\
\hline PML risk & 99 & 75 \\
\hline Pregnancy plan & 21 & 16 \\
\hline Inefficacy & 7 & 5.3 \\
\hline Adverse events & 4 & 3 \\
\hline Cancer & 1 & 0.75 \\
\hline
\end{tabular}

$A R R$ annualized relapse rate, EDSS expanded disability status scale, NTZ natalizumab, PML progressive multifocal leukoencephalopathy, $Q 1$ first quartile, $Q 3$ third quartile

* Unless otherwise stated

Interestingly, no evidence of disease reactivation was observed in the rituximab and AHSCT groups.

Table 2 describes the frequency of disease reactivation according to the different treatment strategies. Table 3 reports the univariate analysis of the different treatments used; for this analysis only groups with a consistent number of patients were included. Interestingly, disease reactivation in the first-line therapy group was not different than in the therapy-free group.

Worsening disability was observed in patients who were therapy free compared to those who restarted DMDs. In fact, at 1 year of follow-up the median EDSS increased from 4.0 (EDSS score at NTZ initiation) to 5.5 (EDSS score 12 months after NTZ discontinuation) in the therapy-free group. A median increase of less than one point EDSS was seen in the treated groups.

\section{Predictors of Reactivation Risk}

Predictors of disease reactivation were the number of NTZ infusions and the duration of the washout period. In particular, a long washout period correlated with MS reactivation at $3\left(\chi^{2} 8.1\right.$, degrees of freedom [DF] 2, $P=0.03), 9\left(\chi^{2} 8.1, D F 2, P=0.03\right)$, and 12 months $\left(\chi^{2}\right.$ 9.7, DF 2, $\left.P=0.01\right)$ after NTZ discontinuation. A lower number of infusions correlated with disease reactivation at 12 months $\left(\chi^{2} 10.06, \quad D F \quad 2, \quad P=0.01\right)$ and rebound at $6\left(\chi^{2} 7.5, D F 2, P=0.04\right)$ and 12 months $\left(\chi^{2} 8.7, D F 2, P=0.02\right)$ after NTZ interruption. In addition, a correlation between a higher number of relapses in the 2 years before NTZ treatment and clinical reactivation at 12 months after NTZ discontinuation was observed $\left(\chi^{2} 81, \quad D F \quad 5, \quad P=0.01\right)$. This association was also observed for the rebound at 12 months $\left(\chi^{2} 11.4, D F 5, P=0.004\right)$. The univariate statistical model did not stratify according to the different therapeutic strategies used. With a multivariate model, no significant differences were observed. Nevertheless, the best predictor of disease reactivation was the number of relapses in the 
Table 2 Clinical/radiological reactivation and rebound in different treatments after NTZ discontinuation

\begin{tabular}{lcccr}
\hline Therapy & Total population & Clinical reactivation & Radiological reactivation & Rebound \\
\hline Therapy-free & $28(37)$ & $59.5(22)$ & $51.4(18)$ & $21.6(8)$ \\
NTZ & $6.8(9)$ & $11(1)$ & $0(0)$ & $0(0)$ \\
FTY & $43.2(57)$ & $17.5(10)$ & $23.6(13)$ & $7(4)$ \\
First-line therapies & $12.1(16)$ & $68.7(11)$ & $28.6(4)$ & $12.5(2)$ \\
Immunosuppressive treatment & $3(4)$ & $25(1)$ & $33.3(1)$ & $25(1)$ \\
Rituximab & $5.3(7)$ & $0(0)$ & $0(0)$ & $0(0)$ \\
AHSCT & $1.5(2)$ & $0(0)$ & $0(0)$ & $0(0)$ \\
\hline
\end{tabular}

Values are presented as percentage $(N)$. More clinical and radiological reactivation was observed in therapy-free group and first-line therapy group. One patient treated with immunosuppressive therapy (mitoxantrone) has developed rebound with a strong clinical and radiological reactivation. Only one patient out of nine who restarted NTZ therapy presented a radiological reactivation. Ten patients (17.5\%) who switched to FTY showed a clinical reactivation and 13 patients (23.6\%) showed a radiological reactivation. No evidence of disease reactivation was observed in rituximab and AHSCT groups AHSCT autologous hematopoietic stem cell transplant, FTY fingolimod, NTZ natalizumab

two years preceding the introduction of NTZ (clinical activity before NTZ, hazard ratio $[\mathrm{HR}]=1.43)$.

In addition, relapse risk was different between treatments. A lower risk of relapses was found in patients treated with NTZ $(\mathrm{HR}=0.29)$ or FTY $(\mathrm{HR}=0.45)$ than in those treated with first-line therapies $(H R=1.6)$. Therefore, second-line therapies demonstrated superiority in preventing disease reactivation after NTZ discontinuation (Table 4 and Fig. 2).

\section{DISCUSSION}

NTZ discontinuation is a frequent clinical practice, mainly as part of the PML risk management strategy. However, NTZ suspension has been associated with a significant disease return often presenting with severe clinical and radiological manifestations. This retrospective study of clinical practice observed patients with MS after NTZ discontinuation to determine the frequency of disease reactivation and to compare the effect of different treatments in reducing this risk.

Our results showed that disease reactivation occurred in more than half of patients who discontinue NTZ and it is most frequent in the 6-9 months following the last infusion of NTZ. It is known from the literature that the concentration of NTZ progressively decreases within 3 months, and changes induced on the immune system can be detected until 6 months after discontinuation [29]. Accordingly, we found a peak of clinical reactivation in month 6 after discontinuation and this activity decreased gradually until 1 year of follow-up. Similarly, we observed greater radiological activity in months 6-9, with a peak at month 9 after NTZ discontinuation. Our study confirms literature data, since the majority of our patients showed a substantial return to their pre-NTZ disease activity [6-8]. However, we found that $30 \%$ of patients developed a rebound activity, and this phenomenon is more frequent if compared 
Table 3 Univariate analysis of different treatments used

\begin{tabular}{|c|c|c|c|c|}
\hline \multirow[t]{2}{*}{ Treatment } & \multirow[t]{2}{*}{ Clinical reactivation (\%) } & \multicolumn{3}{|c|}{ Statistical test } \\
\hline & & $\overline{\chi^{2}}$ & $D F$ & $P$ value \\
\hline Therapy-free & 59.5 & & & \\
\hline First-line therapy & 68.7 & 1.8 & 1 & 0.2 \\
\hline FTY & 17.5 & 37.7 & 1 & $<0.0001$ \\
\hline NTZ & 11 & 51.5 & 1 & $<0.0001$ \\
\hline \multirow[t]{2}{*}{ Treatment } & \multirow{2}{*}{$\begin{array}{l}\text { Radiological } \\
\text { reactivation (\%) }\end{array}$} & \multicolumn{3}{|c|}{ Statistical test } \\
\hline & & $\overline{\chi^{2}}$ & $D F$ & $P$ value \\
\hline Therapy-free & 51.4 & & & \\
\hline First-line therapy & 28.6 & 10.8 & 1 & 0.002 \\
\hline FTY & 23.6 & 165 & 1 & 0.0001 \\
\hline NTZ & 0 & - & - & - \\
\hline \multirow[t]{2}{*}{ Treatment } & \multirow[t]{2}{*}{ Rebound (\%) } & \multicolumn{3}{|c|}{ Statistical test } \\
\hline & & $\overline{\chi^{2}}$ & $D F$ & $P$ value \\
\hline Therapy-free & 21.6 & & & \\
\hline First-line therapy & 12.5 & 2.9 & 1 & 0.13 \\
\hline FTY & 7 & 8.7 & 1 & 0.006 \\
\hline NTZ & 0 & - & - & - \\
\hline \multicolumn{5}{|c|}{$\begin{array}{l}\text { In the univariate analysis, we have excluded groups with few patients (rituximab, immunosuppressive therapy, AHSCT). } \\
\text { The other therapeutic groups (first-line therapy, FTY, NTZ) have been compared to the therapy-free group. Analysis } \\
\text { showed an association between second-line therapy (FTY and NTZ) and a lower clinical and radiological reactivation, and } \\
\text { rebound }\end{array}$} \\
\hline
\end{tabular}

with literature data, probably due to our operative definition as a communal definition has not yet been established [15].

Some studies have identified predictive variables of disease activity for patients discontinuing NTZ: A higher pre-NTZ disease activity and long washout period are factors predicting reactivation [6, 27]. Also in our analysis we confirmed that a higher number of relapses in the 2 years before NTZ treatment and a long washout period were correlated to MS reactivation and rebound. These data indicate that an alternative treatment should be promptly resumed mainly in patients with high disease activity the year before initiating NTZ. In addition, we observed also a correlation between a lower number of infusions of NTZ and clinical reactivation and rebound. The association with short-term use of NTZ has been suggested by few authors, but has not been proven $[14,16]$.

The second aim of our analysis was to evaluate the effects of different therapeutic strategies to reduce disease activity after NTZ interruption. We have observed that second-line therapies were greater for 
Table 4 Multivariate analysis

\begin{tabular}{lccl}
\hline Variable & HR & 95\% CI & $\boldsymbol{P}$ value \\
\hline Clinical activity before NTZ & 1.43 & $0.7-2.9$ & 0.31 \\
Infusions NTZ & 0.99 & $0.9-1.0$ & 0.66 \\
Washout period & 1.0 & $0.9-1.0$ & 0.618 \\
DMDs & & & 0.019 \\
$\begin{array}{l}\text { First-line therapies vs. } \\
\quad \text { therapy-free }\end{array}$ & 1.6 & $0.4-5.3$ & 0.43 \\
FTY vs. therapy-free & 0.45 & $0.1-1.2$ & 0.13 \\
NTZ vs. therapy-free & 0.29 & $0.03-2.3$ & 0.24 \\
\hline
\end{tabular}

The relapse risk was estimated through a Cox regression model adjusted for: number of infusions, relapses in the 2 years before NTZ, washout period between NTZ and new therapy, therapeutic strategies after NTZ (no therapy, first-line therapies, FTY, NTZ); we have excluded groups with few patients (rituximab, immunosuppressive therapy, AHSCT). Value of HR is different between first-line therapy and second-line therapies

AHSCT autologous hematopoietic stem cell transplant, $C I$ confidence interval, DMDs disease-modifying drugs, FTY fingolimod, $H R$ hazard ratio, NTZ natalizumab

preventing disease reactivation compared to none treatment or to first-line therapies. The ineffectiveness of first-line therapies has been confirmed in numerous published data and in clinical experience $[17,19,23]$.

In our analysis, nine patients were retreated with NTZ after its withdrawal. These patients had discontinued treatment because they had a positivity to Stratify test (STRATIFY Gen1); but when STRATIFY Gen2 test showed a low anti-JCV antibodies index [30], these patients reconsidered to restart NTZ therapy, also justified by a recurrence of disease activity.

FTY is a second-line therapy and may be a therapeutic option, especially if it is started early after NTZ discontinuation [19, 21, 27, 31]. However, FTY did not abolish disease activity risk and indeed in our cohort we confirmed a clinical reactivation in 10 out of 57 patients

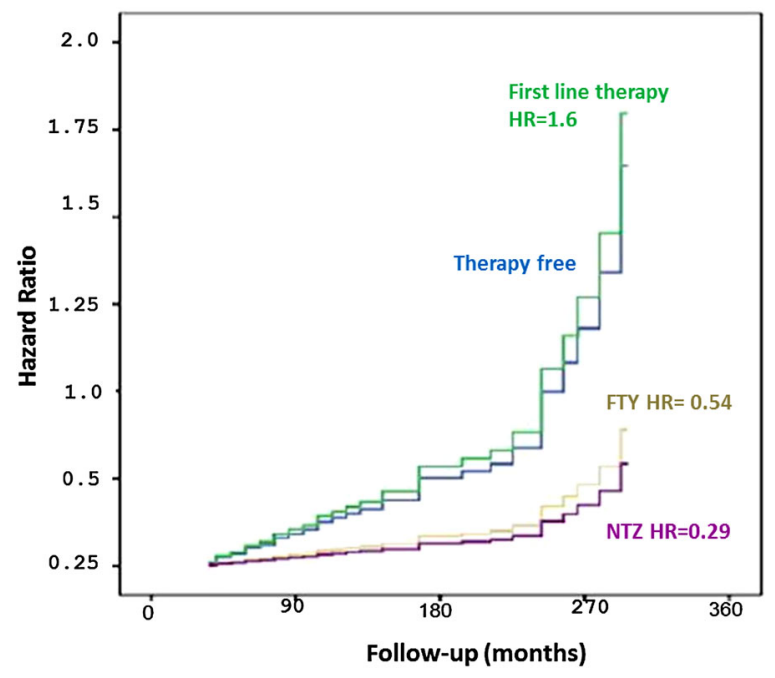

Fig. 2 The relapse risk estimated between therapeutic strategies at 12 months after NTZ discontinuation. Green line first-line therapy group. Blue line therapy-free group. Purple line NTZ group. Yellow line FTY group. The HR values for the groups were as follows: first-line therapy $=1.6 ; \mathrm{NTZ}=0.29 ; \mathrm{FTY}=0.45$. The $\mathrm{HR}$ value of first-line therapy group was similar to $H R$ value of therapy-free group. The relapse risk was lower in patients treated with NTZ or FTY. FTY fingolimod, HR hazard ratio, $N T Z$ natalizumab

(17.5\%) and radiological reactivation in 13 out 55 patients $(23.6 \%)$.

Patients with an aggressive inflammatory clinical course have been treated with immunosuppressive agents (cyclophosphamide or mitoxantrone), AHSCT, and rituximab; no patients were treated with alemtuzumab as it was not available at the time of study.

The use of immunosuppressive agents may be a strategy for patients with aggressive disease, but no definite indication can be done because of our sample size (only 4 patients).

Interesting data were detected in a few patients treated with rituximab (7 patients): no clinical or radiological activity was observed. The median washout period between NTZ suspension and rituximab was 3.8 months (range 1.5-7.8 months). They were patients 
with an active disease but had refused AHSCT and had disagreed to the use of cyclophosphamide as they were young women and wanted to plan a future pregnancy. We choose rituximab treatment because literature data suggest rituximab is effective in patients with aggressive relapsing-remitting MS; however, its use is off-label. We are aware that a larger sample size and longer follow-up are needed to confirm this preliminary result [32].

We do not know the PML risk in patients treated with rituximab after NTZ discontinuation. PML risk restricted to rituximab is difficult to establish; in literature, PML during rituximab therapy was described in patients treated previously with a broad spectrum of immunosuppressive agents [33-35].

In our analysis, patients treated with rituximab were from the MS center of Orbassano. All of these patients underwent MRI examination at NTZ discontinuation and just before the first infusion of rituximab in order to exclude the risk of a clinical undetectable PML.

Two patients were switched to AHSCT; one patient switched to AHSCT 4.5 months after NTZ discontinuation, the other patient switched after 5.6 months. They had very aggressive disease with high radiological and clinical activity before NTZ treatment and after suspension; in particular, one patient experienced one clinical and radiological reactivation in the first month after NTZ discontinuation, the other patient in the third month after suspension. The choice of this therapy strategy was due to evidence of efficacy in patients with very aggressive relapsing-remitting MS [36, 37].

Limitations of our investigation include the low number of patients, the retrospective design, and the lack of complete radiological data. To verify our retrospective results, a prospective and randomized study with a larger sample should be performed; nevertheless, such a study is unlikely as there is not an optimal therapeutic strategy after NTZ discontinuation and therefore therapy of patients is always personalized. As such, retrospective studies are the best methodology today to help neurologists in the management of treatment in NTZ discontinuation period.

\section{CONCLUSIONS}

NTZ interruption represents a current challenge in the optimization and tailoring of MS treatment. Our study confirms the risk of MS reactivation and rebound after NTZ suspension and indicate that an alternative treatment should be promptly resumed mainly in patients with a previous very active disease course. There are no established guidelines for the choice of treatment in patients who discontinue NTZ, but our results suggest the use of second-line treatment. Following this strategy it is mandatory to balance the risk-benefit ratio at the single patient level with a shared-decision strategy, including all the aspects of the patient's life.

\section{ACKNOWLEDGMENTS}

No funding or sponsorship was received for this study or publication of this article. All named authors meet the International Committee of Medical Journal Editors (ICMJE) criteria for authorship for this manuscript, take responsibility for the integrity of the work as a whole, and have given final approval for the version to be published. 
Disclosures. Marianna Lo Re has received travel expenses from Biogen, Novartis, and Teva. Marco Capobianco has received speaking honoraria and/or consultant fees from Biogen, Merck-Serono, Novartis, Teva, Genzyme, and Almirall. Paolo Ragonese has received travel expenses or honoraria for consultancy from Merck Serono, Biogen, Novartis, Sanofi Genzyme, and Teva Pharma. Sabrina Realmuto has received travel expenses from Biogen and Sanofi. Simona Malucchi has received speaking honoraria and/or consultant fees from Biogen, Merck-Serono, Novartis, Teva, and Genzyme. Paola Berchialla has nothing to disclose. Giuseppe Salemi has received travel expenses or honoraria for consultancy from Merck Serono, Biogen, Novartis and Sanofi. Antonio Bertolotto has received honoraria for serving in the scientific advisory boards of Almirall, Bayer, Biogen, and Genzyme with approval by the Director of AOU San Luigi University Hospital, and has received speaker honoraria from Biogen, Genzyme, Novartis, and Teva; his institution has received grant support from Bayer, Biogen, Merck, Novartis, and Teva from the Italian Multiple Sclerosis Society, Fondazione Ricerca Biomedica ONLUS, and San Luigi ONLUS.

Compliance with Ethics Guidelines. Ethical approval was not necessary due to the retrospective plan of the study, according to internal ethical board rules.

Open Access. This article is distributed under the terms of the Creative Commons Attribution-NonCommercial 4.0 International License (http://creativecommons.org/licenses/ by-nc/4.0/), which permits any noncommercial use, distribution, and reproduction in any medium, provided you give appropriate credit to the original author(s) and the source, provide a link to the Creative Commons license, and indicate if changes were made.

\section{REFERENCES}

1. Polman $\mathrm{CH}, \mathrm{O}^{\prime}$ Connor PW, Havrdova E, et al. A randomized, placebo-controlled trial of natalizumab for relapsing multiple sclerosis. N Engl J Med. 2006;354:899-910.

2. Rudick RA, Stuart WH, Calabresi PA, et al. Natalizumab plus interferon beta 1-a for relapsing multiple sclerosis. N Engl J Med. 2006;354:911-23.

3. Baldwin KJ, Hogg JP. Progressive multifocal leukoencephalopathy in patients with multiple sclerosis. Curr Opin Neurol. 2013;26:318-3223.

4. Berger JR, Aksamit AJ, Clifford DB, et al. PML diagnostic criteria. Neurology. 2013;80:1430-8.

5. Sorensen S, Bertolotto A, Edan G, et al. Risk stratification for progressive multifocal leukoencephalopathy in patients treated with natalizumab. Mult Scler. 2012;18(2):143-52.

6. Fox RJ, Cree BA, De Seze J, et al. MS disease activity in RESTORE: a randomized 24-week natalizumab treatment interruption study. Neurology. 2014;82:1491-8.

7. O'Connor PW, Goodman A, Kappos L, et al. Disease activity return during natalizumab treatment interruption in patients with multiple sclerosis. Neurology. 2011;76:1858-65.

8. Berger JR, Centonze D, Comi G, et al. Considerations on discontinuing natalizumab for the treatment of multiple sclerosis. Ann Neurol. 2010;68(3):409-10.

9. Sangalli F, Moiola L, Ferrè L, et al. Long-term management of natalizumab discontinuation in a large monocentric cohort of multiple sclerosis patients. Mult Scler Relat Disord. 2014;3:520-6.

10. Killestein J, Vennegoor A, Strijbis EM, et al. Natalizumab drug holiday in multiple sclerosis: poorly tolerated. Ann Neurol. 2010;68:392-5.

11. Kaufman MD, Lee R, Norton HJ. Course of relapsing remitting multiple sclerosis before, during and after natalizumab. Mult Scler. 2011;17:490-4.

12. Borriello G, Prosperini L, Marinelli F, et al. Observations during an elective interruption of natalizumab treatment: a post-marketing study. Mult Scler. 2011;17:372-5. 
13. Borriello G, Prosperini L, Mancinelli C, et al. Pulse monthly steroids during an elective interruption of natalizumab: a post-marketing study. Eur J Neurol. 2012;19:783-7.

14. Vellinga MM, Castelijns JA, Barkhof F, et al. Post withdrawal rebound increase in T2 lesional activity in natalizumab treated MS patients. Neurology. 2008;70:1150-1.

15. Miravalle A, Jensen R, Kinkel RP. Immune reconstitution inflammatory syndrome in patients with multiple sclerosis following cessation of natalizumab therapy. Arch Neurol. 2011;68(2): 186-91.

16. Schiess N, Calabresi PA. Natalizumab bound to rebound? Neurology. 2009;72:392-3.

17. Havla J, Gerdes LA, Meinl I, et al. De-escalation from natalizumab in multiple sclerosis: recurrence of disease activity despite switching to glatiramer acetate. J Neurol. 2011;9(258):1665-9.

18. West TW, Cree BA. Natalizumab dosage suspension: are we helping or hurting? Ann Neurol. 2010;68(3):395-9.

19. Cohen M, Maillart E, Tourbah A, et al. Switching from natalizumab to fingolimod in multiple sclerosis: a french prospective study. JAMA Neurol. 2014;71:436-41.

20. Magraner MJ, Coret F, Navarre A, et al. Pulsed steroids followed by glatiramer acetate to prevent inflammatory activity after cessation of natalizumab therapy: a prospective, 6-month observational study. J Neurol. 2011;258:1805-11.

21. Laroni A, Brogi D, Milesi V, et al. Early switch to fingolimod may decrease the risk of disease recurrence after natalizumab interruption. Mult Scler. 2012;19(9):1236-7.

22. Jokubaitis V, Li V, Kalincik T, et al. Fingolimod after natalizumab and the risk of short-term relapse. Neurology. 2014;82:1204-11.

23. Iaffaldano P, Lucisano G, Pozzilli $C$, et al. Fingolimod versus interferon beta/glatiramer acetate after natalizumab suspension in multiple sclerosis. Brain. 2015;138:3275-86.

24. Daelman L, Maitrot A, Maarouf A, et al. Severe multiple sclerosis reactivation under fingolimod 3 months after natalizumab withdrawal. Mult Scler. 2012;11(18):1647-9.

25. Centonze D, Rossi S, Rinaldi F, Gallo P. Severe relapses under Fingolimod treatment prescribed after natalizumab. Neurology. 2012;79:2004-6.
26. Rinaldi F, Seppi D, Calabrese M, et al. Switching therapy from natalizumab to fingolimod in relapsing-remitting multiple sclerosis: clinical and magnetic resonance imaging findings. Mult Scler. 2012;18:1640.

27. Kappos L, Radue EW, Comi G. TOFINGO study group. Switching from natalizumab to fingolimod: a randomized, placebo-controlled study in RRMS. Neurology. 2015;85:29-40.

28. Capobianco M, di Sapio A, Malentacchi M, et al. No impact of current therapeutic strategies on disease reactivation after natalizumab discontinuation: a comparative analysis of different approaches during the first year of natalizumab discontinuation. Eur J Neurol. 2015;22(3):585-7.

29. Stüve O, Marra CM, Jerome $K R$, et al. Immune surveillance in multiple sclerosis patients treated with natalizumab. Ann Neurol. 2006;59:743-7.

30. Pavlina T, Subramanyam M, Bloomgren G, et al. Anti-JC virus antibody levels in serum or plasma further define risk of natalizumab associated progressive multifocal leukoencephalopathy. Ann Neurol. 2014;76:802-12.

31. Havla J, Tackenberg B, Hellwig K, et al. Fingolimod reduces recurrence of disease activity after natalizumab withdrawal in multiple sclerosis. J Neurol. 2013;260:1382-7.

32. Hauser SL, Waubant E, Arnold DL, et al. B-cell depletion with rituximab in relapsing-remitting multiple sclerosis. N Engl J Med. 2008;358:676-88.

33. Castillo-Trivino T, Braithwaite D, Bacchetti P, et al. Rituximab in relapsing and progressive forms of multiple sclerosis: a systematic review. PLoS One. 2013;8(7):e66308.

34. Tuccori M, Focosi D, Blandizzi C, et al. Inclusion of rituximab in treatment protocols for non-Hodgkin's lymphomas and risk for progressive multifocal leukoencephalopathy. Oncologist. 2010;15:1214-9.

35. Clifford DB, Ances B, Costello C, et al. Rituximab-associated progressive multifocal leukoencephalopathy in rheumatoid arthritis. Arch Neurol. 2011;68:1156-64.

36. Pfender N, Saccardi R, Martin R. Autologous hematopoietic stem cell transplantation as a treatment option for aggressive multiple sclerosis. Curr Treat Option Neurol. 2013;15:270-80.

37. Mancardi GL, Sormani MP, Gualandi F, et al. Autologous hematopoietic stem cell transplantation in multiple sclerosis: a phase II trial. Neurology. 2015;84(10):981-8. 